\title{
Heat Kernels and Super Determinants of Laplace Operators on Super Riemann Surfaces ${ }^{\star}$
}

\author{
Kenichiro Aoki \\ Joseph Henry Laboratories of Physics, Princeton University, Princeton, New Jersey 08544, USA
}

\begin{abstract}
Heat kernels of Laplacians on superfields of arbitrary tensor weight on super Riemann surfaces are constructed, and are used to compute the super determinants of these operators in terms of the Selberg super zeta function.
\end{abstract}

\section{Introduction}

In the perturbation theory of closed superstrings, scattering amplitudes are formulated in terms of expectation values of products of vertex operators on compact surfaces, summed over the surfaces and the location of these operators on these surfaces. The summation is carried out over the distinct topologies (genus) with a measure for each topology. In the Polyakov formulation of the superstring amplitudes [1], one sums over the distinct geometries and embeddings of the surfaces into the target spacetime and divides by the volume of the invariance group of the measure. Weyl rescalings belong to the invariance group only when the target spacetime has the critical dimension. We will work with the type II superstrings in flat spacetime having critical dimension $d=10$.

In the superspace approach, the invariances are super reparametrizations, super Weyl rescalings, local Lorentz rotations and Poincaré transformations of the target spacetime. One finds the measure over supermoduli space to be a product of superdeterminants of Laplacians acting on scalar and vector superfields on the super Riemann surface [2-5].

In the component formalism, the invariances are reparametrizations, local supersymmetry transformations, Weyl rescalings, super Weyl transformations, local Lorentz rotations and spacetime Poincaré symmetries. All the odd supermoduli can be integrated out to obtain the measure as a density on moduli space. In the Wess-Zumino gauge, the measure reduces to a product of determinants of Laplacians acting on spin $0,1 / 2,1,3 / 2$ fields on the Riemann surface and the Pfaffian of an operator depending on the zero modes of the Dirac operator $[6,7]$.

\footnotetext{
* Work supported in part by the National Science Foundation grant, NSF-PHY-80-19754
} 
The superspace approach has the advantage of treating the local supersymmetry transformations as a special case of super reparametrizations and also unifies Weyl and super Weyl rescalings, at the cost of having to deal with super analysis. The local supersymmetry transformations are complicated when one stays in the Wess-Zumino gauge and the symmetry not manifest. Therefore, we will work in the superspace formalism, retaining manifest super reparametrization invariance throughout this paper.

We first construct the heat kernels for Laplacians on superfields of arbitrary tensor weight on the super upper half plane with the super Poincaré geometry, which has constant curvature. This generalizes to superspace some of the work of Fay [11], in which he constructed the heat kernels for Laplacians on tensors and spinors on the upper half plane. The use of the transformation properties of the Laplacians under the isometry group of the super upper half plane, $\operatorname{OSp}(2 \mid 1, R) /\{ \pm 1\}$, and the analogue of polar coordinates with simple transformation properties under the above group, considerably simplifies the construction. We will also make use of the spectral decomposition of the Maass Laplacians in our construction. We next derive the heat kernel on the compact super Riemann surface of genus $g \geqq 2$. We represent the super Riemann surface as the quotient space of the super upper half plane by a super Fuchsian group, thereby parametrizing the supermoduli by constant curvature super geometries. The heat kernel on the super Riemann surface is constructed by the method of images and its super trace is computed with the help of the Selberg trace formula.

The super determinant is calculated using the zeta function obtained as the Mellin transform of the trace of the heat kernel. We find the super determinant of the Laplacian expressed in terms of values of the Selberg super zeta function at half integer points and a factor depending only on the genus of the surface and the weight of the superfield. We find that the square of the Laplacian is not positive semidefinite, contrary to what one would naively expect. The calculation of the super determinant follows the lines of [8]. The measure for genus $g \geqq 2$ was calculated in the component formalism in $[6,7]$ and in $[2-5]$ in superspace. The determinants of Laplacians for tensors and spinors of arbitrary weight on compact Riemann surfaces of genus $g \geqq 2$ were calculated in [8] with the help of the heat kernels of these operators derived by Fay [11], parametrizing the moduli by constant curvature metrics (see also $[9,10]$ ).

Our work was motivated by the theory of superstrings. However, the heat kernel and the super determinant of the Laplacian, and the Selberg super zeta function of the super Riemann surface generalize the corresponding concepts of the Riemann surface, and are of interest on its own right.

Section 2 gives a description of the super geometry, super uniformization and tensors on super Riemann surfaces. The heat kernel for the Laplacian on the super upper half plane is constructed in Sect. 3. Section 4 contains the Selberg trace formula and we compute the super determinants in Sect. 5.

After this work was completed, we became aware of the work of Baranov, Manin, Frolov and Schwarz [12], where the ratios of the super determinants are calculated in terms of the Selberg super zeta function by somewhat different methods. Our results are consistent with theirs. The super determinants in the 
superstring measure were also considered by Martinec in [13], where instead, uniformization by Schottky groups was used to parametrize supermoduli space.

\section{Super Geometry, Super Uniformization and Tensors}

Super Geometry. Super geometry is specified by the choice of the zweibein $\left(E_{M}{ }^{A}\right)$ and the connection $\left(\Omega_{M}\right)$ corresponding to 20 real superfield degrees of freedom in $2 \mid 2$ real dimensions ${ }^{1}$. (We follow $[3,14,15]$.) The connection $\left(\Omega_{M}\right)$ belongs to the algebra of the local Lorentz group and we will restrict the super geometry by taking the local Lorentz group to be $U(1)$. A Lorentz covariant derivative acting on a superfield of tensor weight $n$ is defined as,

$$
D_{A}^{(n)}=E_{A}{ }^{M}\left(\partial_{M}+i n \Omega_{M}\right) .
$$

Torsion $\left(T_{A B}{ }^{C}\right)$ and curvature $\left(R_{A B}\right)$ are defined by the following relation:

$$
\left[D_{A}, D_{B}\right]=-T_{A B}^{C} D_{C}^{(n)}+i n R_{A B} .
$$

Tensor weights are defined by the transformation properties under the local Lorentz group as follows.

$$
\begin{gathered}
\zeta \mapsto e^{i \varphi} \zeta, \\
T^{(n)} \mapsto e^{i n \varphi} T^{(n)} .
\end{gathered}
$$

$V^{+}, V^{\zeta}$ have weight $1 / 2,1$ for example.

We impose the following standard torsion constraints [17] up to trivial rescaling:

$$
\begin{aligned}
T_{++{ }^{\bar{\zeta}}} & =T_{--^{\bar{\zeta}}=-2,} T_{\alpha \beta}{ }^{c}=0 \text { (otherwise) }, \\
T_{\alpha \beta}{ }^{\gamma} & =T_{a b}{ }^{c}=0 .
\end{aligned}
$$

The torsion constraints are not only super reparametrization invariant and local Lorentz covariant, but also super Weyl invariant as will be shown below. We note that the only nonzero constraints are imposed on Lorentz scalars. These give 14 equations leaving 6 real superfield degrees of freedom. This means we cannot even specify $E_{+}{ }^{M}$ and $E_{-}{ }^{M}$ independently [2].

We can equivalently specify the super geometry by the covariant derivatives $\left(D_{A}^{(n)}\right)$, defining the zweibein and the connection by the relation (2.1). In this paper, this latter approach will be taken and we will not make use of the explicit expressions of the zweibein and the connection. As remarked in the preceding paragraph, the super geometry is uniquely determined by the choice of $D_{+}^{(0)}$ and $D_{-}^{(0)}$, which have to be chosen to comply with the torsion constraints (2.4).

1 We use complex coordinates and $A, B, C \ldots(M, N, P \ldots)$ will denote Lorentz (Einstein) indices,

$$
\begin{array}{lll}
A=a, \alpha, & a=\zeta, \bar{\zeta}, & \alpha=+,-, \\
M=m, \mu, & m=z, \bar{z}, & \mu=\theta, \bar{\theta} .
\end{array}
$$

Latin(Greek) letters denote vector(spinor) indices 
In flat superspace, covariant derivatives with Lorentz indices are given by,

$$
\hat{D}_{\alpha}: \quad \hat{D}_{+}^{(0)}=\frac{\partial}{\partial \theta}+\theta \frac{\partial}{\partial z}, \quad \hat{D}_{-}^{(0)}=\frac{\partial}{\partial \bar{\theta}}+\bar{\theta} \frac{\partial}{\partial \bar{z}} .
$$

Using the definition of torsion and curvature (2.2) and the torsion constraints (2.4), other covariant derivatives in flat super geometry can be worked out as in [18] to be,

$$
\begin{aligned}
\hat{D}_{\alpha}: \quad \hat{D}_{\zeta}^{(n)} & =\frac{\partial}{\partial z}=\left(\hat{D}_{+}^{(n)}\right)^{2}, \quad \hat{D}_{\bar{\zeta}}^{(n)}=\frac{\partial}{\partial \bar{z}}, \\
\hat{T}_{A B}{ }^{C} & =\hat{R}_{A B}=\hat{\Omega}_{M}=0, \\
\text { except } \quad \hat{T}_{++}{ }^{\zeta} & =\hat{T}_{--} \bar{\zeta}=-2 .
\end{aligned}
$$

We will use the notation $\hat{D}_{A}$ for flat superspace covariant derivatives unless otherwise stated.

Given one super geometry $\left(\hat{D}_{A}\right)$ (not necessarily flat), one can obtain another super geometry $\left(D_{A}\right)$ by a super Weyl rescaling [17]. Namely, we define $D_{\alpha}$ by,

$$
\begin{aligned}
D_{\alpha}^{(n)}: & D_{+}^{(n)}=e^{-(n+1 / 2) \Phi} \hat{D}_{+}^{(n)} e^{n \Phi}, \\
& D_{-}^{(n)}=e^{(n-1 / 2) \Phi} \hat{D}_{-}^{(n)} e^{-n \Phi},
\end{aligned}
$$

where $\Phi$ is an arbitrary real scalar superfield. (To be precise, $D_{+}^{(0)}$ in (2.2) acting on a scalar superfield gives $E_{ \pm}{ }^{M} \Omega_{M}$ using torsion constraints (2.4), which in turn gives (2.7)). This transformation leaves the torsion constraints (2.4) unchanged. As before, other covariant derivatives, torsion and curvature can be derived by repeated use of (2.2) and (2.4). For instance,

$$
D_{+}^{(n-1 / 2)} D_{+}^{(n)}=D_{\zeta}^{(n)}+\frac{i}{2} n R_{++}
$$

gives $R_{++}=0$ and,

$$
\begin{aligned}
& D_{\zeta}^{(n)}=e^{-\Phi}\left(\hat{D}_{\zeta}-\hat{D}_{+} \Phi \hat{D}_{+}+n \hat{D}_{\zeta} \Phi\right), \\
& D_{\bar{\zeta}}^{(n)}=e^{-\Phi}\left(\hat{D}_{\bar{\zeta}}-\hat{D}_{-} \Phi \hat{D}_{-}-n \hat{D}_{\bar{\zeta}} \Phi\right) .
\end{aligned}
$$

We also derive,

$$
R_{+-}=e^{-\Phi}\left(\hat{R}_{+-}+2 i \hat{D}_{+} \hat{D}_{-} \Phi\right)
$$

The super reparametrization invariant volume element transforms as follows under a super Weyl transformation:

$$
E d^{2} z d^{2} \theta=e^{\Phi} \hat{E} d^{2} z d^{2} \theta,
$$

where $E=\operatorname{sdet}\left(E_{M}{ }^{A}\right)$ and sdet denotes the super determinant defined in [19], for example.

Super Uniformization. Let us recall the uniformization theory of ordinary Riemann surfaces. The group of conformal automorphisms of the complex projective line (the Riemann sphere) is $S L(2, C) /\{ \pm 1\}$ (Möbius transformations). The action on 
the projective line is given by (in inhomogeneous coordinates),

$$
\begin{gathered}
\gamma=\left(\begin{array}{ll}
a & b \\
c & d
\end{array}\right) \in S L(2, C) /\{ \pm 1\}, \\
z \mapsto \gamma z=\frac{a z+b}{c z+d} .
\end{gathered}
$$

The subgroup of $S L(2, C) /\{ \pm 1\}$ that fixes the real line is $S L(2, R) /\{ \pm 1\}$, the group of conformal automorphisms of the upper half plane $H=\{z \in C \mid \mathscr{I} z>0\}$. One defines the Poincare geometry on the upper half plane $H$ by the metric,

$$
g_{z \bar{z}}=g_{\bar{z} z}=\frac{1}{2 y^{2}}, \quad g_{z z}=g_{\bar{z} \bar{z}}=0, \quad z=x+i y .
$$

This geometry has constant negative curvature -1 . The line element

$$
d s^{2}=\frac{d z d \bar{z}}{y^{2}}
$$

and the volume element

$$
\frac{d x d y}{y^{2}}
$$

are invariant under the action of $S L(2, R) /\{ \pm 1\}$.

Every compact Riemann surface $M_{g}$ of genus $g \geqq 2$ is isometric to $H / \Gamma$ with the Poincare geometry. The Fuchsian group $\Gamma$ is a subgroup of $S L(2, R) /\{ \pm 1\}$ generated by $2 g$ elements $\left\{A_{i}, B_{i}, i=1, \ldots g\right\}$ acting properly discontinuously on the upper half plane $H$ with all its elements hyperbolic $(\mid$ trace $\mid>2)$. The generators satisfy the condition,

$$
A_{1} B_{1} A_{1}^{-1} B_{1}^{-1} A_{2} B_{2} A_{2}^{-1} B_{2}^{-1} \ldots A_{g} B_{g} A_{g}^{-1} B_{g}^{-1}=1 .
$$

Inequivalent Fuchsian groups span the moduli space, which has $6 g-6$ real dimensions.

The complex super projective line is the set of points $(u, v, \phi)(u, v$ even elements of complex Grassmann algebra, $\phi$ odd) not all its body ${ }^{2}$ of the coordinates simultaneously zero, and $(u, v, \phi)$ is identified with $(\lambda u, \lambda v, \lambda \phi)$ for $\lambda$ even. It has complex dimension 1|1. A superconformal automorphism of the complex super projective line must be a homogeneous map in homogeneous coordinates. Since it induces a conformal automorphism of its body, the complex projective line, it must be linear. It can be expressed as,

$$
\left(\begin{array}{l}
u \\
v \\
\phi
\end{array}\right) \mapsto \gamma\left(\begin{array}{l}
u \\
v \\
\phi
\end{array}\right) \quad \gamma=\left(\begin{array}{lll}
a & b & \alpha \\
c & d & \beta \\
\delta & \varepsilon & A
\end{array}\right)
$$

\footnotetext{
${ }^{2}$ We will refer to the non-nilpotent and the nilpotent part of the Grassmann algebra as the body and the soul respectively
} 
or using inhomogeneous coordinates $(z, \theta)=(u / v, \phi / v)$,

$$
z \mapsto \frac{a z+b+\alpha \theta}{c z+d+\beta \theta}, \quad \theta \mapsto \frac{\delta z+\varepsilon+A \theta}{c z+d+\beta \theta} .
$$

A map, $(z, \theta) \mapsto(\tilde{z}, \tilde{\theta})$ is called superconformal when $\tilde{z}$ and $\tilde{\theta}$ are functions of $z$ and $\theta$, analytic in $z$ and not a function of $\bar{\theta}$ satisfying $[14,20]$,

$$
\hat{D}_{+} \tilde{z}-\tilde{\theta} \hat{D}_{+} \tilde{\theta}=0 \text {. }
$$

This condition ensures that the flat superspace derivatives $\hat{D}_{+}$transform homogeneously,

$$
\hat{D}_{+}=\left(\hat{D}_{+} \tilde{\theta}\right) \hat{\tilde{D}}_{+}
$$

After choosing the normalization sdet $\gamma=1$, the condition of superconformality is equivalent to the following condition on the automorphism $\gamma$ of (2.14),

$$
{ }^{t} \gamma J \gamma=J
$$

where

$$
J=\left(\begin{array}{rrr}
0 & -1 & 0 \\
1 & 0 & 0 \\
0 & 0 & 1
\end{array}\right) . \quad{ }^{t} \gamma=\left(\begin{array}{rrr}
a & c & \delta \\
b & d & \varepsilon \\
-\alpha & -\beta & A
\end{array}\right) .
$$

This means the group of superconformal automorphisms of the complex super projective line is $O S p(2 \mid 1, C) /\{ \pm 1\} . O S p(2 \mid 1)$ is the super extension of $S L(2) \simeq S p(2)$ defined by (2.18) and $\operatorname{dim} \operatorname{OSp}(2 \mid 1)=3 \mid 2$.

The super upper half plane $s H=\{Z=(z, \theta) \mid \mathscr{I} z>0\}$ is a complex supermanifold of dimension 1|1 having the upper half plane $H$ as its body. (Positivity is defined by the positivity of its body.) The subgroup of $\operatorname{OSp}(2 \mid 1, C) /\{ \pm 1\}$ which fixes the super real line $\{(z, \theta) \mid z=\bar{z}, \theta=\bar{\theta}\}$ is $\operatorname{OSp}(2 \mid 1, R) /\{ \pm 1\}$, the group of superconformal automorphisms of $s H$ that extends to the super real line and the point at infinity. The behavior at infinity is defined using the superconformal transition function $(z, \theta)=(-1 / \tilde{z},-\tilde{\theta} / \tilde{z})$ in the neighborhood of $\tilde{z}=0$.

Super Poincare geometry $\left(D_{A}\right)$ on $s H$ is defined by a super Weyl transform of the flat super geometry $\left(\hat{D}_{A}\right)$, by the following choice of the scalar superfield in (2.7),

$$
e^{\Phi}=\frac{2 i}{z-\bar{z}-\theta \bar{\theta}}
$$

giving a constant curvature super geometry $R_{+-}=-1$ using (2.9). The line element $d s^{2}=E^{\zeta} E^{\bar{\zeta}}$, where

$$
E^{A}=d z^{M} E_{M}{ }^{A} \quad E^{\zeta}=e^{\Phi} \hat{E}^{\zeta}=\frac{2 i d Z}{z-\bar{z}-\theta \bar{\theta}} \quad d Z=d z+\theta d \theta
$$

is invariant under $\operatorname{OSp}(2 \mid 1, R) /\{ \pm 1\}$. Our choice of the super Weyl rescaling in (2.19) gives the volume element (2.10) as,

$$
E d^{2} z d^{2} \theta=\frac{-4}{z-\bar{z}-\theta \bar{\theta}} d x d y d^{2} \theta
$$


normalized so that it gives the Poincare geometry volume element (2.12) when integrating a function having no nilpotent parts. The super jacobian of the $\operatorname{OSp}(2 \mid 1, R) /\{ \pm 1\}$ transformation can be calculated to be,

$$
\operatorname{sdet} \frac{\partial(\gamma z, \gamma \theta)}{\partial(z, \theta)}=\frac{1}{v_{\gamma}(c z+d+\beta \theta)} .
$$

This shows the volume element (2.21) is invariant under $\operatorname{OSp}(2 \mid 1, R) /\{ \pm 1\}$ transformation, also using (2.23) below.

We will restrict ourselves to super Riemann surfaces having contractible soul (DeWitt topology [16]). Any super Riemann surface $s M_{g}$ of this type having compact body is isometric to $s H / s \Gamma[14,15]$. $s \Gamma$ is a subgroup of $\operatorname{OSp}(2 \mid 1, R) /\{ \pm 1\}$ called the super Fuchsian group. The body of the first two rows and columns form the Fuchsian group corresponding to the body $M_{g}$ underlying $s M_{g}$. The super Fuchsian group is isomorphic to this Fuchsian group due to the condition of having no topology in the soul, so that the super Fuchsian group $s \Gamma$ is again generated by $2 g$ elements satisfying the generator condition (2.13). The supermoduli space, the space of inequivalent super Fuchsian groups, is $(6 g-6) \mid(4 g-4)$ real dimensional, taking into account the generator condition (2.13) and the freedom of one overall conjugation.

Any element of the super Fuchsian group $s \Gamma$ is diagonalizable in $\operatorname{OSp}(2 \mid 1, R) /$ $\{ \pm 1\}$. We choose the representatives of $s \Gamma$ in $\operatorname{OSp}(2 \mid 1, R) /\{ \pm 1\}$ to have positive super-trace (sum of even diagonals minus odd diagonals). The sign is defined by the sign of its body.

where

$$
s \Gamma \ni \gamma=\left(\begin{array}{ccc}
a & b & \alpha \\
c & d & \beta \\
\delta & \varepsilon & v_{\gamma}(1-\alpha \beta)
\end{array}\right) \underset{v_{\gamma}= \pm 1}{\operatorname{osp(2|1,R)/\{ \pm 1\} }\left(\begin{array}{ccc}
e^{l_{\gamma} / 2} & 0 & 0 \\
0 & e^{-l_{\gamma} / 2} & 0 \\
0 & 0 & v_{\gamma}
\end{array}\right)}
$$

due to the invariance of the super trace under conjugation.

The sign of $v_{\gamma}$ and $(c z+d+\beta \theta)$ depend on our choice of representatives, but the product $v_{\gamma}(c z+d+\beta \theta)$ is well defined for any element in $\operatorname{OSp}(2 \mid 1, R) /\{ \pm 1\}$. $\{v\}$ is the multiplier system [11], which incorporates the spin structure naturally into the definition of a super Riemann surface.

A spin structure on the Riemann surface is defined by a choice of the lifting of the Fuchsian group $\Gamma \subset S L(2, R) /\{ \pm 1\}$ to a group $\tilde{\Gamma} \subset S L(2, R)$. A multiplier system $\{\chi\}$ is the description of this choice in terms of a homomorphism of $\tilde{\Gamma}$ into $\{ \pm 1\}$ such that $\chi(-1)=-1$. Due to our convention of taking positive trace, $v_{\gamma}=\chi_{\gamma} \operatorname{sign}(\operatorname{tr} \gamma)$ and $v$ in general is not multiplicative (sign of trace is not). We note that $v_{\gamma \delta}=v_{\gamma} v_{\delta}$ if $\gamma \delta=\delta \gamma$.

Tensors and Laplacians on super Riemann Surfaces. To define the space of tensors on the super Riemann surface, we need the transformation law of the following 
combination of two superspace coordinates $Z_{a}, Z_{b}$,

$$
\begin{aligned}
z_{a b} & =z_{a}-z_{b}-\theta_{a} \theta_{b}, \\
z_{a b} \stackrel{\gamma}{\mapsto} \gamma\left(z_{a b}\right) & =\gamma z_{a}-\gamma z_{b}-\gamma \theta_{a} \gamma \theta_{b} \\
& =\frac{z_{a b}}{w_{a} w_{b}}, \\
w_{a} & =v_{\gamma}\left(c z_{a}+d+\beta \theta_{a}\right), \ldots \text { etc. }
\end{aligned}
$$

where $\gamma$ was given in (2.14). The infinitesimal form of (2.23) gives,

$$
d(\gamma Z)=\frac{d Z}{w^{2}}, \quad \text { where } \quad d Z=d z+\theta d \theta .
$$

We define the space of tensors $\left\{f(Z) d Z^{m} \otimes d \bar{z}^{n}\right\}$. In particular, we define the space $\mathscr{H}_{n, v}$ for $n$ half integer as,

$$
\begin{aligned}
\mathscr{H}_{n, v}= & \left\{f(Z) d Z^{n / 2} \otimes d \bar{Z}^{-n / 2}\right\} \\
= & \left\{f \mid f(\gamma Z)=v_{\gamma}^{2 n}\left(\frac{c z+d+\beta \theta}{c \bar{z}+d+\beta \bar{\theta}}\right)^{n} f(Z), \gamma \in s \Gamma\right\} \\
& f \text { :function on } s H .
\end{aligned}
$$

Another simple tensor $\Theta_{a b c}$ we will need later is defined as,

$$
\begin{aligned}
& \Theta_{a b c}=\theta_{a} z_{b c}+\theta_{b} z_{c a}+\theta_{c} z_{a b}+\theta_{a} \theta_{b} \theta_{c}, \\
& \Theta_{a b c} \stackrel{\gamma}{\mapsto} \gamma\left(\Theta_{a b c}\right)=\frac{\Theta_{a b c}}{w_{a} w_{b} w_{c}} .
\end{aligned}
$$

Since $\operatorname{OSp}(2 \mid 1, R) /\{ \pm 1\}$ has real dimensions $3 \mid 2$ and acts effectively on the super upper half plane $s H$, from 2 points $Z_{1}, Z_{2}$ on $s H$, we can construct 1 Grassmann even and 2 odd invariants. Together with an angular variable $\varphi, z_{a b}$ and $\Theta_{a b c}$ allow us to define a super hyperbolic coordinate system on $s H$ as follows:

$$
\begin{aligned}
\cosh r\left(Z_{1}, Z_{2}\right) & =1-2 \frac{z_{12} z_{\overline{1} \overline{2}}}{z_{1 \overline{1}} z_{2 \overline{2}}}, \quad e^{2 i \varphi\left(Z_{1}, Z_{2}\right)}=\frac{z_{12} z_{\overline{1} 2}}{z_{1 \overline{2}} z_{\overline{1} \overline{2}}}, \\
\Delta & =\frac{\Theta_{12 \overline{2}}}{\left(z_{12} z_{2 \overline{2}} z_{\overline{2} 1}\right)^{1 / 2}}, \quad \bar{\Delta}=\frac{\Theta_{\overline{1} \overline{2} 2}}{\left(z_{\overline{1} \overline{2}} z_{\overline{2} 2} z_{2 \overline{1}}\right)^{1 / 2}}, \\
z_{\overline{1}} & =\bar{z}_{1}, \theta_{\overline{1}}=\bar{\theta}_{1}, \ldots \text { etc. }
\end{aligned}
$$

$\cosh r$ is the integrated form of the line element (2.20). We note that $\varphi$ is not $\operatorname{OSp}(2 \mid 1, R) /\{ \pm 1\}$ invariant whereas $r, \Delta, \bar{\Delta}$ and $\partial / \partial \varphi$ are.

The superspace derivative transforms under $\operatorname{OSp}(2 \mid 1, R) /\{ \pm 1\}$ as (cf. (2.17)),

$$
\begin{aligned}
& \hat{D}_{+} \stackrel{\gamma}{\mapsto} w \hat{D}_{+}, \\
& w=v_{\gamma}(c z+d+\beta \theta)=\left(\hat{D}_{+}(\gamma \theta)\right)^{-1} .
\end{aligned}
$$


From the definitions in (2.7), (2.8) and (2.19), we derive the covariant derivatives,

$$
\begin{aligned}
& D_{+}^{(n)}=\left(\frac{z-\bar{z}-\theta \bar{\theta}}{2 i}\right)^{1 / 2}\left(\hat{D}_{+}-n \frac{\theta-\bar{\theta}}{z-\bar{z}-\theta \bar{\theta}}\right), \\
& D_{-}^{(n)}=\left(\frac{z-\bar{z}-\theta \bar{\theta}}{2 i}\right)^{1 / 2}\left(\hat{D}_{-}+n \frac{\theta-\bar{\theta}}{z-\bar{z}-\theta \bar{\theta}}\right), \\
& D_{\zeta}^{(n)}=\frac{1}{2 i}\left((z-\bar{z}-\theta \bar{\theta}) \frac{\partial}{\partial z}+(\theta-\bar{\theta}) \hat{D}_{+}-n\right), \\
& D_{\bar{\zeta}}^{(n)}=\frac{1}{2 i}\left((z-\bar{z}-\theta \bar{\theta}) \frac{\partial}{\partial \bar{z}}+(\theta-\bar{\theta}) \hat{D}_{-}-n\right) .
\end{aligned}
$$

Using (2.23), (2.27) and (2.28) we verify,

$$
\begin{aligned}
& \mathscr{H}_{-n-1 / 2, v} \stackrel{D_{-(n)}^{\longleftarrow}}{\longleftarrow} \mathscr{H}_{-n, v} \stackrel{D_{+}^{(n)}}{\longrightarrow} \mathscr{H}_{-n+1 / 2, v}, \\
& \mathscr{H}_{-n-1, v} \stackrel{D_{\zeta}^{(n)}}{\longleftarrow} \mathscr{H}_{-n, v} \stackrel{D_{\xi}^{(n)}}{\longrightarrow} \mathscr{H}_{-n+1, v} .
\end{aligned}
$$

From the above equations (2.28) we see that $D_{+}^{(n)}$ and $D_{-}^{(n)}$ are, loosely speaking, the square roots of the Maass operators, $K_{-n}, L_{-n}$,

$$
K_{-n}=(z-\bar{z}) \frac{\partial}{\partial z}-n, \quad L_{-n}=(\bar{z}-z) \frac{\partial}{\partial \bar{z}}+n .
$$

$D_{+} D_{-}^{(n)}$ is the Laplacian on the super Riemann surface mapping the space $\mathscr{H}_{-n, v}$ into itself.

We define a nondegenerate inner product on $\mathscr{H}_{-n, v}$ as follows using the measure in (2.21):

$$
\langle\Phi \mid \Psi\rangle=\int E d^{2} z d^{2} \theta \bar{\Phi} \Psi .
$$

The Laplacian $D_{+} D_{-}^{(n)}$ is hermitian with respect to this inner product, but we do not know whether the operator is diagonalizable. Even if it were, the space $\mathscr{H}_{-n, v}$ contains isotropic vectors and the eigenvalues for these vectors need not be real.

\section{The Heat Kernel}

In this section we construct the heat kernel for the operator $\Delta_{n}=4\left(D_{+} D_{-}^{(n)}\right)^{2}$ on the super upper half plane $s H$. We use the square of the Laplacian $\Delta_{n}$ rather than the Laplacian $D_{+} D_{-}^{(n)}$ itself, following Martinec [21], who constructed heat kernels of these operators in flat superspace to calculate the conformal anomalies of the super determinants in the superstring measure. $\Delta_{n}$ is roughly the Maass Laplacian, and we expect it to be better behaved than the Laplacian itself. Due to the remarks at the end of the previous section, even the square of the Laplacian $\Delta_{n}$ need not be positive semidefinite, and will turn out not to be (cf. the end of this section). 
From the definition of the covariant derivatives $D_{ \pm}^{(n)}$ in (2.28),

$$
\begin{aligned}
\Delta_{n}= & z_{1 \overline{1}}^{2} \frac{\partial}{\partial z} \frac{\partial}{\partial \bar{z}}+z_{1 \overline{1}} \theta_{1 \overline{1}}\left(\frac{\partial}{\partial z} \hat{D}_{-}+\frac{\partial}{\partial \bar{z}} \hat{D}_{+}\right) \\
& +(2 n+1) z_{1 \overline{1}} \hat{D}_{-} \hat{D}_{+}-n z_{1 \overline{1}}\left(\frac{\partial}{\partial z}+\frac{\partial}{\partial \bar{z}}\right)+2 n^{2} \theta_{1 \overline{1}}\left(\hat{D}_{-}+\hat{D}_{+}\right)-n^{2}, \\
\theta_{1 \overline{1}}= & \theta_{1}-\bar{\theta}_{1}
\end{aligned}
$$

with the notation of Sect. 2, and all derivatives act on variables with suffix 1 .

The defining relations for the heat kernel $\hat{G}_{n}^{t}\left(Z_{1}, Z_{2}\right)$ are,

$$
\begin{aligned}
& \left(\partial_{t}+\Delta_{n}\right) \hat{G}_{n}^{t}\left(Z_{1}, Z_{2}\right)=0 \quad(t>0), \\
& \hat{G}_{n}^{t}\left(Z_{1}, Z_{2}\right) \stackrel{t \rightarrow+0}{\longrightarrow} E^{-1} \delta\left(x_{1}-x_{2}\right) \delta\left(y_{1}-y_{2}\right) \delta^{2}\left(\theta_{1}-\theta_{2}\right), \\
& E=\frac{-4}{z_{1 \overline{1}}} \\
& \delta^{2}\left(\theta_{1}-\theta_{2}\right)=\left(\theta_{1}-\theta_{2}\right)\left(\bar{\theta}_{1}-\bar{\theta}_{2}\right) .
\end{aligned}
$$

Following [11], we conjugate $\Delta_{n}$ to make an operator $\tilde{\Delta}_{n}$ which is $\operatorname{OSp}(2 \mid 1, R) /\{ \pm 1\}$ invariant due to (2.23), (2.27) and (2.28),

$$
\tilde{\Delta}_{n}=\left(\frac{z_{1 \overline{2}}}{z_{2 \overline{1}}}\right)^{-n} \Delta_{n}\left(\frac{z_{1 \overline{2}}}{z_{2 \overline{1}}}\right)^{n} .
$$

Using the super hyperbolic coordinate system introduced in (2.26), $\tilde{\Delta}_{n}$ can be expressed in terms of $\operatorname{OSp}(2 \mid 1, R) /\{ \pm 1\}$ invariant variables $r, \Delta, \tilde{\Delta}$, and operators $\partial / \partial r, \partial / \partial \varphi, \partial / \partial \Delta, \partial / \partial \bar{\Delta}$

Recalling that the volume element $(2.21)$ is $\operatorname{OSp}(2 \mid 1, R) /\{ \pm 1\}$ invariant, the superspace delta function and hence the heat kernel for $\tilde{\Delta}_{n}$ can be expressed in $\operatorname{OSp}(2 \mid 1, R) /\{ \pm 1\}$ invariant variables as,

$$
G_{n}^{t}\left(Z_{1}, Z_{2}\right)=g_{n}^{t}(r)+i \Delta \bar{\Delta} h_{n}^{t}(r) .
$$

It satisfies

$$
\begin{aligned}
& \left(\partial_{t}+\tilde{\Delta}_{n}\right) G_{n}^{t}\left(Z_{1}, Z_{2}\right)=0 \quad(t>0), \\
& \frac{1}{\sinh r} G_{n}^{t}\left(Z_{1}, Z_{2}\right) \stackrel{t \rightarrow+0}{\longrightarrow}-\frac{1}{2 \pi} \delta(\cosh r-1) i \Delta \bar{\Delta},
\end{aligned}
$$

where we have taken into account the jacobian,

$$
E^{-1} \operatorname{sdet}\left(\frac{\partial(\cosh r, \varphi, \Delta, \bar{\Delta})}{\partial(x, y, \theta, \bar{\theta})}\right)=-\sinh r .
$$

The relation between the heat kernels $\hat{G}_{n}^{t}\left(Z_{1}, Z_{2}\right)$ and $G_{n}^{t}\left(Z_{1}, Z_{2}\right)$ is

$$
\hat{G}_{n}^{t}\left(Z_{1}, Z_{2}\right)=\left(\frac{z_{1 \overline{2}}}{z_{2 \overline{1}}}\right)^{n} G_{n}^{t}\left(Z_{1}, Z_{2}\right) .
$$

Equation (3.2) is now reduced to coupled heat equations involving only $r$ and $t$, 
which we will now attempt to solve. Changing variables in the Laplacian (3.1) the equations now become,

$$
\begin{aligned}
\left\{\frac{\partial}{\partial t}-\left[\frac{\partial^{2}}{\partial r^{2}}+\left(\frac{1}{\sinh r}-2 n \tanh \frac{r}{2}\right) \frac{\partial}{\partial r}+n^{2}\left(1+\frac{2}{\cosh r+1}\right)\right]\right\} g_{n}^{t}(r) \\
=\frac{2(2 n+1)}{\sinh r} h_{n}^{t}(n), \\
\left\{\frac{\partial}{\partial t}-\left[\frac{\partial^{2}}{\partial r^{2}}+\left(-\frac{1}{\sinh r}+2 n \tanh \frac{r}{2}\right)+\frac{\cosh r}{\sinh ^{2} r}+\frac{2\left(n+n^{2}\right)}{\cosh r+1}+n^{2}\right]\right\} h_{n}^{t}(r) \\
+2 n \frac{\sinh r}{\cosh r+1}\left[\frac{\partial^{2}}{\partial r^{2}}+\frac{1}{\sinh r} \frac{\partial}{\partial r}-\frac{n}{\cosh r+1}-n^{2}\right] g_{n}^{t}(r)=0,
\end{aligned}
$$

with the condition that

$$
g_{n}^{t}(r) \stackrel{t \rightarrow+0}{\longrightarrow} 0, \quad \frac{1}{\sinh r} h_{n}^{t}(r) \stackrel{t \rightarrow+0}{\longrightarrow}-\frac{1}{2 \pi} \delta(\cosh r-1) .
$$

Since the equations do not seem to decouple, we will use the following trick. From Eq. (3.2) in the coordinates on $s H$, we derive the equation for the body $\hat{g}_{n}^{t}\left(z_{1}, z_{2}\right)$ of the heat kernel $\hat{G}_{n}^{t}\left(Z_{1}, Z_{2}\right)$. We then construct this body $\hat{g}_{n}^{t}\left(z_{1}, z_{2}\right)$ by spectral methods and hence deduce $g_{n}^{t}(r)$ in (3.4). Using the heat equation (3.6), we compute $h_{n}^{t}(r)$ and we show that this together with $g_{n}^{t}(r)$ satisfies the rest of the equations, (3.7) and (3.8).

The body of $\hat{G}_{n}^{t}\left(Z_{1}, Z_{2}\right)$ is coupled via (3.4) to $O\left(\theta_{1} \bar{\theta}_{1}\right)$ term. So we expand $\hat{G}_{n}^{t}\left(Z_{1}, Z_{2}\right)$ as

$$
\hat{G}_{n}^{t}\left(Z_{1}, Z_{2}\right)=\hat{g}_{n}^{t}\left(z_{1}, z_{2}\right)+\frac{\theta_{1} \bar{\theta}_{1}}{z_{1}-\bar{z}_{1}} \hat{F}_{n}^{t}\left(z_{1}, z_{2}\right)+\text { terms involving } \theta_{2}, \bar{\theta}_{2} .
$$

From (3.5), the relation between $g_{n}^{t}(r)$ and $\hat{g}_{n}^{t}\left(z_{1}, z_{2}\right)$ is,

$$
\text { the body of } g_{n}^{t}(r)=\left(\frac{z_{1}-\bar{z}_{2}}{z_{2}-\bar{z}_{1}}\right)^{-1} \hat{g}_{n}^{t}\left(z_{1}, z_{2}\right) \text {. }
$$

Equation (3.2) reduces to

$$
\begin{aligned}
& {\left[\frac{\partial}{\partial t}-\left(D_{-n}+n^{2}\right)\right] \hat{g}_{n}^{t}\left(z_{1}, z_{2}\right)+(2 n+1) \hat{F}_{n}^{t}\left(z_{1}, z_{2}\right)=0} \\
& \quad(2 n+1) D_{-n} \hat{g}_{n}^{t}\left(z_{1}, z_{2}\right) \\
& \quad+\left[\frac{\partial}{\partial t}-\left(D_{-n}+(n+1)^{2}\right)\right] \hat{F}_{n}^{t}\left(z_{1}, z_{2}\right)=y_{1}^{2} \delta(t) \delta\left(x_{1}-x_{2}\right) \delta\left(y_{1}-y_{2}\right),
\end{aligned}
$$

where

$$
D_{-n}=y^{2}\left(\frac{\partial^{2}}{\partial x^{2}}+\frac{\partial^{2}}{\partial y^{2}}\right)+2 i n y \frac{\partial}{\partial x}
$$


is the Maass laplacian. Together, they yield an equation for $\hat{g}_{n}^{t}\left(z_{1}, z_{2}\right)$,

$$
\begin{aligned}
& \left\{\left[\frac{\partial}{\partial t}-\left(D_{-n}+(n+1)^{2}\right)\right]\left[\frac{\partial}{\partial t}-\left(D_{-n}+n^{2}\right)\right]-(2 n+1)^{2} D_{-n}\right\} \hat{g}_{n}^{t}\left(z_{1}, z_{2}\right) \\
& \quad=-(2 n+1) y_{1}^{2} \delta(t) \delta\left(x_{1}-x_{2}\right) \delta\left(y_{1}-y_{2}\right) .
\end{aligned}
$$

We make use of the following eigenfunctions for the Maass laplacian $D_{-n}$, $\left(\psi_{p, k}\right)_{p>0, k \in R}$.

$$
\begin{aligned}
D_{-n} \psi_{p, k}(x, y) & =-\left(\frac{1}{4}+p^{2}\right) \psi_{p, k}(x, y), \\
\psi_{p, k}(x, y) & =\frac{\left|\Gamma\left(\frac{1}{2}-\mu+i p\right)\right|}{2} \sqrt{\frac{p \sinh 2 \pi p}{\pi^{3}|k|}} e^{i k x} W_{\mu, i p}(2|k| y), \\
\mu & =-\operatorname{sign}(k) n, \quad z=x+i y .
\end{aligned}
$$

$W_{\mu, i p}(\omega)$ is the Whittacker function and $\left(\psi_{p, k}\right)$ is normalized so that,

$$
\int_{-\infty}^{\infty} d x \int_{0}^{\infty} \frac{d y}{y^{2}} \bar{\psi}_{p, k}(x, y) \psi_{p^{\prime}, k^{\prime}}(x, y)=\delta\left(p-p^{\prime}\right) \delta\left(k-k^{\prime}\right)
$$

Using the Laplace transform in $t$, the solution to (3.10) is,

$$
\hat{g}_{n}^{t}\left(z_{1}, z_{2}\right)=-(2 n+1) \int_{c+i R} \frac{d s}{2 \pi i} \int_{-\infty}^{\infty} d k \int_{0}^{\infty} d p \frac{e^{s t} \psi_{p, k}\left(x_{1}, y_{1}\right) \bar{\psi}_{p, k}\left(x_{2}, y_{2}\right)}{\left(s-\left(\frac{1}{2}+n+i p\right)^{2}\right)\left(s-\left(\frac{1}{2}+n-i p\right)^{2}\right)} .
$$

Using partial fractions and integrating over $s$,

$$
\begin{aligned}
\hat{g}_{n}^{t}\left(z_{1}, z_{2}\right)= & -\int_{-\infty}^{\infty} d k \int_{0}^{\infty} \frac{d p}{2 i p} e^{-t p^{2}+t(n+1 / 2)^{2}} \psi_{p, k}\left(x_{1}, y_{1}\right) \bar{\psi}_{p, k}\left(x_{2}, y_{2}\right) \\
& \cdot\left[e^{2 i p(n+1 / 2) t}-e^{-2 i p(n+1 / 2) t}\right] .
\end{aligned}
$$

From the work of Fay [11], we know the heat kernel $\hat{h}_{n}^{t}\left(z_{1}, z_{2}\right)$ for the Maass Laplacian $D_{-n}$ as,

$$
\begin{aligned}
\hat{h}_{n}^{t}\left(z_{1}, z_{2}\right) & =\int_{-\infty}^{\infty} d k \int_{0}^{\infty} d p e^{-\left(p^{2}+1 / 4\right) t} \psi_{p, k}\left(x_{1}, y_{1}\right) \bar{\psi}_{p, k}\left(x_{2}, y_{2}\right) \\
& =\left(\frac{z_{1}-\bar{z}_{2}}{z_{2}-\bar{z}_{1}}\right)^{n} \frac{e^{-t / 4}}{(4 \pi t)^{3 / 2}} \int_{r}^{\infty} \frac{d b b e^{-b^{2} / 4 t}}{\sqrt{2(\cosh b-\cosh r)}} T_{2 n}\left(\frac{\cosh b / 2}{\cosh r / 2}\right),
\end{aligned}
$$

where $T_{k}(x)$ denotes the $k^{\text {th }}$ Chebyshev polynomial. We make use of the following integral representation of the Whittacker function [26]:

$$
\begin{aligned}
& W_{\mu, i p}\left(2|k| y_{1}\right) W_{\mu, i p}\left(2|k| y_{2}\right) \\
& =2|k| \frac{\left(y_{1} y_{2}\right)^{i p+1 / 2} e^{-|k|\left(y_{1}+y_{2}\right)}}{\Gamma(1-2 \mu)} \int_{0}^{\infty} d t e^{-2|k| t} t^{-2 \mu}\left[\left(y_{1}+t\right)\left(y_{2}+t\right)\right]^{\mu-1 / 2-i p} \\
& \quad \cdot F\left(\frac{1}{2}-\mu+i p, \frac{1}{2}-\mu+i p ; 1-2 \mu ; \frac{t\left(y_{1}+y_{2}+t\right)}{\left(y_{1}+t\right)\left(y_{2}+t\right)}\right)
\end{aligned}
$$


and for the hypergeometric function [27],

$$
F(\alpha, \beta ; \gamma ; \theta)=\frac{\Gamma(\gamma)}{\Gamma(\beta) \Gamma(\gamma-\beta)} \int_{0}^{1} d w w^{\beta-1}(1-w)^{\gamma-\beta-1}(1-w \theta)^{-\alpha} .
$$

Integrating over $p, k$ and comparing $\hat{g}_{n}^{t}\left(z_{1}, z_{2}\right)$ in (3.11) and $\hat{h}_{n}^{t}\left(z_{1}, z_{2}\right)$ in (3.12) we deduce $\hat{g}_{n}^{t}\left(z_{1}, z_{2}\right)$ and use (3.9) to obtain,

$$
g_{n}^{t}(r)=\frac{-1}{2 \pi^{3 / 2} t^{1 / 2}} \int_{r}^{\infty} \frac{d b e^{-b^{2} / 4 t} \sinh (n+1 / 2) b}{\sqrt{2(\cosh b-\cosh r)}} T_{2 n}\left(\frac{\cosh b / 2}{\cosh r / 2}\right) .
$$

We can now use (3.6) to calculate $h_{n}^{t}(r)$ as,

$$
h_{n}^{t}(r)=-\frac{\sinh r / 2}{4 \pi^{3 / 2} t^{3 / 2}} \int_{r}^{\infty} \frac{d b b e^{-b^{2} / 4 t} \cosh n b}{\sqrt{2(\cosh b-\cosh r)}} T_{2 n+1}\left(\frac{\cosh b / 2}{\cosh r / 2}\right)-n \tanh \frac{r}{2} g_{n}^{t}(r) .
$$

Using the definitions (3.3), (3.4), (3.5), we obtain the final expression for the heat kernel $\hat{G}_{n}^{t}\left(Z_{1}, Z_{2}\right)$ on $s H$ as,

$$
\hat{G}_{n}^{t}\left(Z_{1}, Z_{2}\right)=\left(\frac{z_{1 \overline{2}}}{z_{2 \overline{1}}}\right)^{n}\left(g_{n}^{t}(r)+i \Delta \bar{\Delta} h_{n}^{t}(r)\right)
$$

Even though we simply have to differentiate $g_{n}^{t}(r)$ to derive $h_{n}^{t}(r)$ as in (3.14), the calculation is reasonably involved and we give the outline in Appendix A.

We see immediately that the condition (3.8) on the behavior of $g_{n}^{t}(r)$ and $h_{n}^{t}(r)$ as $t$ goes to zero is satisfied from the comparison with the heat kernel $\hat{h}_{0}^{t}(r)$ for the scalar Laplacian $D_{0}=y^{2}\left(\left(\partial^{2} / \partial x^{2}\right)+\left(\partial^{2} / \partial y^{2}\right)\right)$,

$$
\hat{h}_{0}^{t}(r)=\frac{e^{-t / 4}}{(4 \pi t)^{3 / 2}} \int_{r}^{\infty} \frac{d b b e^{-b^{2} / 4 t}}{\sqrt{2(\cosh b-\cosh r)}}, \quad \hat{h}_{0}^{t}(r) \stackrel{t \rightarrow+0}{\longrightarrow} \frac{1}{2 \pi} \delta(\cosh r-1) .
$$

Equations (3.13), (3.14) and (3.15) complete the construction of the heat kernel $\hat{G}_{n}^{t}\left(Z_{1}, Z_{2}\right)$ for the operator $\Delta_{n}$ on $s H$.

\section{The Selberg Trace Formula}

From the heat kernel on the super upper half plane $s H$, we construct the heat kernel on the super Riemann surface $s H / s \Gamma$ by the Poincare sum [11],

$$
K_{n}^{t}\left(Z, Z^{\prime}\right)=\sum_{\gamma \in s \Gamma} v_{\gamma}^{2 n}\left(\frac{c \bar{z}^{\prime}+d+\beta \bar{\theta}^{\prime}}{c z^{\prime}+d+\beta \theta^{\prime}}\right)^{n}\left(\frac{z-\gamma \bar{z}^{\prime}-\theta \gamma \overline{\theta^{\prime}}}{\gamma z^{\prime}-\bar{z}-\gamma \theta^{\prime} \bar{\theta}}\right)^{n} G_{n}^{t}\left(Z, \gamma Z^{\prime}\right) .
$$

$(\gamma$ is given in $(2.14))$

As explained in Sect. 2, the multiplier system $v$ is necessary for the expression to be well defined. $K_{n}^{t}\left(Z, Z^{\prime}\right)$ belongs to $\mathscr{H}_{-n, v}, \mathscr{H}_{n, v}^{\prime}$ defined in $(2.25)$ with respect to $Z, Z^{\prime}$ respectively.

$$
\operatorname{Str}\left(e^{-t \Delta_{n}}\right)=\int_{s H / s \Gamma} E d^{2} z d^{2} \theta K_{n}^{t}(Z, Z)=I_{n}^{e}(t)+I_{n}(t)
$$


where $I_{n}^{e}(t)$ and $I_{n}(t)$ are defined as,

$$
\begin{aligned}
& I_{n}^{e}(t)=\int_{s H / s \Gamma} E d^{2} z d^{2} \theta G_{n}^{t}(Z, Z), \\
& I_{n}(t)=\sum_{\substack{\gamma \in S \Gamma \\
\gamma \neq e}} v_{\gamma}^{2 n} \int_{s H / s \Gamma} E d^{2} z d^{2} \theta\left(\frac{c \bar{z}+d+\beta \bar{\theta}}{c z+d+\beta \theta}\right)^{n}\left(\frac{z-\gamma \bar{z}-\theta \gamma \bar{\theta}}{\gamma z-\bar{z}-\gamma \theta \bar{\theta}}\right)^{n} G_{n}^{t}(Z, \gamma Z) .
\end{aligned}
$$

Repartition the sum in $I_{n}(t)$ as follows [22]:

$$
\sum_{\substack{\gamma \in S \Gamma \\ \gamma \neq e}} f(\gamma)=\sum_{[\gamma] \neq e} \sum_{k \in S \Gamma / C_{\Gamma}(\gamma)} f\left(k^{-1} \gamma k\right)
$$

where $C_{\Gamma}(\gamma)=\{k \in s \Gamma \mid k \gamma=\gamma k\}$ is the centralizer of $\gamma$ in $s \Gamma$ and $[\gamma]$ is the conjugacy class represented by $\gamma$ in $s \Gamma$. This leads to

$$
\begin{aligned}
I_{n}(t)= & \sum_{[\gamma] \neq e} \sum_{k \in s \Gamma / C_{\Gamma}^{(\gamma)}} v_{k^{-1} \gamma k}^{2 n} \int_{s H / s \Gamma} E d^{2} z d^{2} \theta\left(\frac{\tilde{c} \bar{z}+\tilde{d}+\tilde{\beta} \bar{\theta}}{\tilde{c} z+\tilde{d}+\tilde{\beta} \theta}\right)^{n} \\
& \cdot\left(\frac{z-k^{-1} \gamma k \bar{z}-\theta k^{-1} \gamma k \bar{\theta}}{k^{-1} \gamma k z-\bar{z}-k^{-1} \gamma k \theta \bar{\theta}}\right)^{n} G_{n}^{t}\left(Z, k^{-1} \gamma k Z\right),
\end{aligned}
$$

where

$$
k^{-1} \gamma k=\left(\begin{array}{ccc}
* & * & * \\
\tilde{c} & \tilde{d} & \tilde{\beta} \\
* & * & *
\end{array}\right) .
$$

Using $\operatorname{OSp}(2 \mid 1, R) /\{ \pm 1\}$ invariance of the volume element $E d^{2} z d^{2} \theta$ and the following formulae:

$$
\begin{aligned}
& (\tilde{c} \bar{z}+\tilde{d}+\tilde{\beta} \bar{\theta})\left(z-k^{-1} \gamma k \bar{z}-\theta k^{-1} \gamma k \bar{\theta}\right) \\
& \quad=(c(k z)+d+\beta(k \theta))(k z-\gamma(k \bar{z})-(k \theta) \gamma(k \bar{\theta})), \quad v_{k^{-1} \gamma k}=v_{\gamma}, \\
& G_{n}^{t}\left(Z, Z^{\prime}\right)=G_{n}^{t}\left(k Z, k Z^{\prime}\right) \quad \text { for } \quad k \in O \operatorname{Sp}(2 \mid 1, R) /\{ \pm 1\},
\end{aligned}
$$

we find

$$
I_{n}(t)=\sum_{[\gamma] \neq e} \sum_{k \in S} \int_{\Gamma / C_{\Gamma^{(\gamma)}}} \int_{k(s H / s \Gamma)} E d^{2} z d^{2} \theta\left(\frac{c \bar{z}+d+\beta \bar{\theta}}{c z+d+\beta \theta}\right)^{n}\left(\frac{z-\gamma \bar{z}-\theta \gamma \bar{\theta}}{\gamma z-\bar{z}-\gamma \theta \bar{\theta}}\right)^{n} G_{n}^{t}(Z, \gamma Z) .
$$

Due to the isomorphism of the super Fuchsian group $s \Gamma$ with the Fuchsian group $\Gamma$, we can determine the structure of $C_{\Gamma}(\gamma)$ using $\Gamma$, which we will identify with $S \Gamma$ in the following discussion. Diagonalizing $\gamma$ in $S L(2, R) /\{ \pm 1\}$ we see that $C_{\Gamma}(\gamma)$ is commutative, and since $\Gamma$ acts properly discontinuously on $H$, there is an element $\delta$ with minimal geodesic length $l_{\delta}$ defined by the relation $\cosh l_{\delta} / 2=$ $\operatorname{tr} \delta(\mathrm{cf} .(2.22)) . C_{\Gamma}(\gamma)$ is a cyclic group generated by this primitive element. (A primitive element in $\Gamma$ is an element which is a positive power only of itself in the group.)

We diagonalize the element $\delta$ in $\operatorname{OSp}(2 \mid 1, R) /\{ \pm 1\}$ as in (2.22),

$$
C_{\Gamma}(\gamma)=\langle\delta\rangle, \quad \delta \sim\left(\begin{array}{ccc}
e^{l_{\delta} / 2} & 0 & 0 \\
0 & e^{-l_{\delta} / 2} & 0 \\
0 & 0 & v_{\delta}
\end{array}\right),
$$


and use the original argument due to Selberg [22],

$$
\bigcup_{k \in s \Gamma / C_{\Gamma}(\gamma)} k(s H / s \Gamma)=s H / C_{\Gamma}(\gamma)=\left\{(x+i y, \theta) \mid 1 \leqq y<e^{l_{\delta}}\right\}
$$

to derive

$$
\begin{aligned}
& I_{n}(t)=\sum_{\gamma \text { prim }} \sum_{p=1}^{\infty} v_{\gamma}^{2 n p} \int_{-\infty}^{\infty} d x \int_{1}^{e^{l_{\gamma}}} d y \int E d^{2} \theta\left(\frac{z-e^{p l_{\nu}} \bar{z}-v_{\gamma}^{p} e^{p l_{\gamma} / 2} \theta \bar{\theta}}{e^{p l_{\gamma} z}-\bar{z}-v_{\gamma}^{p} e^{p l_{\gamma} / 2} \theta \bar{\theta}}\right)^{n} \\
& \cdot G_{n}^{t}\left(z, \theta ; e^{p l_{\gamma} z, v_{\gamma}^{p}} e^{p l_{\gamma} / 2} \theta\right) \text {, }
\end{aligned}
$$

where the first sum is over the primitive conjugacy classes, and $G_{n}^{t}$ was defined in (3.3), (3.13), (3.14).

We proceed to compute $I_{n}(t)$ explicitly. From $(2.26)$ for $\left(Z_{1}, Z_{2}\right)=(Z, \gamma Z)$,

where

$$
\cosh r=\cosh p l_{\gamma}+2 v^{2} \sinh ^{2} \frac{p l_{\gamma}}{2}+\frac{\theta \bar{\theta}}{(z-\bar{z})} 4\left(1+v^{2}\right) \sinh ^{2} \frac{p l_{\gamma}}{2},
$$

$$
\begin{aligned}
v & =\frac{x}{y}, \\
i \Delta \bar{\Delta} & =\frac{\theta \bar{\theta}}{(z-\bar{z})} \frac{4\left(v_{\gamma}^{p} \cosh \frac{p l_{\gamma}}{2}-1\right)}{\sinh r} .
\end{aligned}
$$

Integrating with respect to $\theta$ and $\bar{\theta}$,

$$
\begin{aligned}
I_{n}(t)= & \sum_{\gamma \operatorname{prim}} \sum_{p=1}^{\infty} v_{\gamma}^{2 n p} \int_{-\infty}^{\infty} d x \int_{1}^{e^{l_{\gamma}}} \frac{d y}{y^{2}}\left(\frac{z-e^{p l_{\gamma} \bar{z}}}{e^{p l_{\gamma} z-\bar{z}}}\right)^{n}\left\{g_{n}^{t}\left(r_{0}\right)+4\left(1+v^{2}\right) \frac{\sinh ^{2} \frac{p l_{\gamma}}{2}}{\sinh r_{0}} \frac{\partial}{\partial r_{0}} g_{n}^{t}\left(r_{0}\right)\right. \\
& \left.+n \frac{v_{\gamma}^{p} p^{p l_{\gamma} / 2}\left(z^{2}-\bar{z}^{2}\right)\left(1-e^{p l_{\gamma}}\right)}{\left(z-e^{p l_{\gamma}}\right)\left(e^{p l_{\gamma} z}-\bar{z}\right)} g_{n}^{t}\left(r_{0}\right)+4 \frac{\left(v_{\gamma}^{p} \cosh \frac{p l_{\gamma}}{2}-1\right)}{\sinh r_{0}} h_{n}^{t}\left(r_{0}\right)\right\},
\end{aligned}
$$

where $\cosh r_{0}=\cosh p l_{\gamma}+2 v^{2} \sinh ^{2} p l_{\gamma} / 2$ is the hyperbolic distance. (We will drop the subscript 0 in what follows.) Using

$$
\left(\frac{z-e^{p l_{\gamma} \bar{z}}}{e^{p l_{\gamma} z-\bar{z}}}\right)=\frac{\cosh \frac{p l_{\gamma}}{2}+i v \sinh \frac{p l_{\gamma}}{2}}{\cosh \frac{p l_{\gamma}}{2}-i v \sinh \frac{p l_{\gamma}}{2}}
$$

and integrating over $y$,

$$
I_{n}(t)=\sum_{\gamma \text { prim }} \sum_{p=1}^{\infty} v_{\gamma}^{2 n p} l_{\gamma} \int_{-\infty}^{\infty} d v\left(\frac{\cosh \frac{p l_{\gamma}}{2}+i v \sinh \frac{p l_{\gamma}}{2}}{\cosh \frac{p l_{\gamma}}{2}-i v \sinh \frac{p l_{\gamma}}{2}}\right)^{n}
$$




$$
\begin{gathered}
\left\{g_{n}^{t}(r)+4\left(1+v^{2}\right) \frac{\sinh ^{2} \frac{p l_{\gamma}}{2}}{\sinh r} \frac{\partial}{\partial r} g_{n}^{t}(r)+2 n v_{\gamma}^{p} \sinh \frac{p l_{\gamma}}{2}\right. \\
\left.\cdot \frac{i v}{\cosh ^{2} \frac{p l_{\gamma}}{2}+v^{2} \sinh ^{2} \frac{p l_{\gamma}}{2}} g_{n}^{t}(r)+4 \frac{\left(v_{\gamma}^{p} \cosh \frac{p l_{\gamma}}{2}-1\right)}{\sinh r} h_{n}^{t}(r)\right\}
\end{gathered}
$$

From (3.13), we calculate,

$$
\begin{aligned}
\frac{\partial}{\partial r} g_{n}^{t}(r)= & \frac{-\sinh r}{2 \pi^{3 / 2} t^{1 / 2}} \int_{r}^{\infty} \frac{d b e^{-b^{2} / 4 t}}{\sqrt{2(\cosh b-\cosh r)}}\left\{\frac{n}{2} \frac{\sinh (n+1 / 2) b}{\cosh r / 2 \cosh b / 2} T_{2 n-1}\left(\frac{\cosh b / 2}{\cosh r / 2}\right)\right. \\
& +\left[\left(n+\frac{1}{2}\right) \frac{\cosh (n+1 / 2) b}{\sinh b}-\frac{\sinh (n+1 / 2) b \cosh b}{\sinh ^{2} b}\right. \\
& \left.\left.-\frac{n}{2} \frac{\sinh (n+1 / 2) b}{\cosh ^{2} r / 2}-\frac{b}{2 t} \frac{\sinh (n+1 / 2) b}{\sinh b}\right] T_{2 n}\left(\frac{\cosh b / 2}{\cosh r / 2}\right)\right\} .
\end{aligned}
$$

Using the expressions of $g_{n}^{t}(r), h_{n}^{t}(r)$ in (3.13), (3.14) and changing the order of integration, we arrive at,

$$
\begin{aligned}
& I_{n}(t)=\sum_{\gamma \text { prim }} \sum_{p=1}^{\infty} \frac{l_{\gamma} v_{\gamma}^{2 n p}}{4 \pi^{3 / 2} t^{1 / 2} \sinh \frac{p l_{\gamma}}{2}} \int_{p l_{\gamma}}^{\infty} d b e^{-b^{2} / 4 t} \\
& \left\{(2 n-1) \sinh \left(n+\frac{1}{2}\right) b J_{2 n}-2 n \frac{\sinh (n+1 / 2) b}{\cosh \frac{p l_{\gamma}}{2}}\left(N_{2 n}+N_{2 n}^{\prime}\right)\right. \\
& +4 \sinh ^{2} \frac{p l_{\gamma}}{2}\left[-\left(n+\frac{1}{2}\right) \frac{\cosh (n+1 / 2) b}{\sinh b}+\frac{\sinh (n+1 / 2) b \cosh b}{\sinh ^{2} b}\right. \\
& \left.+\frac{b}{2 t} \frac{\sinh (n+1 / 2) b}{\sinh b}\right]\left(J_{2 n}+K_{2 n}\right)+2 n \frac{\sinh (n+1 / 2) b}{\cosh b / 2} \\
& \cdot\left(N_{2 n-1}^{\prime}-\cosh \frac{p l_{\gamma}}{2} J_{2 n-1}-\sinh \frac{p l_{\gamma}}{2} P_{2 n-1}\right)+\frac{b}{t} \cosh n b N_{2 n+1} \\
& \left.+v_{\gamma}^{p}\left[2 n \sinh \left(n+\frac{1}{2}\right) b N_{2 n}-\frac{b}{t} \cosh \frac{p l_{\gamma}}{2} \cosh n b N_{2 n+1}\right]\right\},
\end{aligned}
$$

where $J_{k}, K_{k}, N_{k}, N_{k}^{\prime}, P_{k}$, are definite integrals involving the $k^{\text {th }}$ Chebyshev polynomial $T_{k}(x)$. They are defined and calculated in Appendix B. Using the results of Appendix B and integrating by parts with respect to $b$, we derive the final form of $I_{n}(t)$

$$
I_{n}(t)=\sum_{\gamma \text { prim }} \sum_{p=1}^{\infty} \frac{l_{\gamma} v_{\gamma}^{2 n p}}{\sqrt{4 \pi t} \sinh \frac{p l_{\gamma}}{2}} e^{-p^{2} l_{\gamma}^{2} / 4 t}\left[\cosh \left(n+\frac{1}{2}\right) p l_{\gamma}-v_{\gamma}^{p} \cosh n p l_{\gamma}\right] .
$$


For the contribution from the identity of $s \Gamma, h_{n}^{t}(r)$ does not contribute and we derive from (3.13) and (4.3),

$$
I_{n}^{e}(t)=-\operatorname{vol}\left(s M_{g}\right) \frac{1}{2 \pi^{3 / 2} t^{1 / 2}} \int_{0}^{\infty} d b e^{-b^{2} / 4 t} \frac{\sinh (n+1 / 2) b}{2 \sinh b / 2} T_{2 n}(\cosh b / 2) .
$$

Using the Gauss-Bonnet theorem with our choice of constant curvature super geometry in (2.21) and the measure (2.19) on $s H$,

$$
\operatorname{vol}\left(s M_{g}\right)=\operatorname{vol}\left(M_{g}\right)=2 \pi\left|\chi\left(M_{g}\right)\right|,
$$

where $\chi\left(M_{g}\right)=2-2 g$ is the Euler character of the underlying manifold $M_{g}$. $T_{2 n}(\cosh b / 2)=\cosh n b$ allows us to find a recursion relation for $I_{n}^{e}(t)$ and derive,

$$
I_{n}^{e}(t)=-\frac{\left|\chi\left(M_{g}\right)\right|}{2} \sum_{k=0}^{2|n|} e^{k^{2} t}
$$

As remarked in Sect. 2, we find that the square of the Laplacian $\Delta_{n}$ is not positive semidefinite, for,

$$
\operatorname{Str}\left(e^{-t \Delta_{n}}\right) \stackrel{t \sim+\infty}{\sim} e^{4 n^{2} t}
$$

\section{Calculation of the Super Determinants}

Since $\Delta_{n}$ is not positive semidefinite, we will calculate the super determinant of $\Delta_{n}+c^{2}$ for $\mathscr{R} c>2|n|$ and analytically continue in $c$, following Sarnak [10]. The super determinant is defined using the $\zeta$ function as,

$$
\begin{aligned}
& \operatorname{sdet}\left(\Delta_{n}+c^{2}\right)=\exp \left(-\frac{\partial \zeta_{n}}{\partial s}(0 ; c)\right) \\
& \zeta_{n}(s ; c)=\operatorname{Str}\left(\left(\Delta_{n}+c^{2}\right)^{-s}\right)=\frac{1}{\Gamma(s)} \int_{0}^{\infty} d t t^{s-1} \operatorname{Str}\left(e^{-t\left(\Delta_{n}+c^{2}\right)}\right)
\end{aligned}
$$

We divide $\zeta_{n}(s ; c)$ into the contributions from the identity and the rest of $s \Gamma$ as follows:

$$
\begin{aligned}
& \zeta_{n}(s ; c)=\zeta_{n}^{e}(s ; c)+\zeta_{n}^{r}(s ; c), \\
& \zeta_{n}^{e}(s ; c)=\frac{1}{\Gamma(s)} \int_{0}^{\infty} d t t^{s-1} e^{-t c^{2}} I_{n}^{e}(t), \\
& \zeta_{n}^{r}(s ; c)=\frac{1}{\Gamma(s)} \int_{0}^{\infty} d t t^{s-1} e^{-t c^{2}} I_{n}(t),
\end{aligned}
$$

where $I_{n}^{e}(t), I_{n}(t)$ are defined in (4.6), (4.5).

$\zeta_{n}^{e}(s ; c)$ is obviously the contribution from the isolated modes and we derive,

$$
\exp \left(-\frac{\partial \zeta_{n}^{e}}{\partial s}(0 ; c)\right)=\left[\prod_{k=0}^{2|n|}\left(c^{2}-k^{2}\right)\right]^{-\left|\chi\left(M_{g}\right)\right| / 2} .
$$

In superstring theory, this factor renormalizes the coupling constant, after setting $c=0$ and removing the zeros. 
To calculate $\zeta_{n}^{r}(s ; c)$, we follow Ray and Singer [28], Fried [23]. Substituting,

$$
t^{s-1}=\frac{1}{\Gamma(1-s)} \int_{0}^{\infty} d \lambda 2(\lambda+c)[\lambda(\lambda+2 c)]^{-s} e^{-\lambda(\lambda+2 c) t}
$$

in (5.2), and after changing the order of integration, we use

$$
\int_{0}^{\infty} d t 2(\lambda+c) e^{-t c^{2}} e^{-\lambda(\lambda+2 c) t} \frac{e^{-p^{2} l_{y}^{2} / 4 t}}{\sqrt{4 \pi t}}=e^{-p l_{y}(\lambda+c)}
$$

to derive

$$
\begin{aligned}
\zeta_{n}^{r}(s ; c)= & \int_{0}^{\infty} \frac{d \lambda[\lambda(\lambda+2 c)]^{-s}}{\Gamma(s) \Gamma(1-s)} \sum_{\gamma \operatorname{prim}} \sum_{p=1}^{\infty} \\
& \cdot \frac{l_{\gamma} v_{\gamma}^{2 n p} e^{p l_{\gamma}(-\lambda-c+1 / 2)}}{\left(e^{p l_{\gamma}}-1\right)} 2\left[\cosh \left(n+\frac{1}{2}\right) p l_{\gamma}-v_{\gamma}^{p} \cosh n p l_{\gamma}\right] .
\end{aligned}
$$

The Selberg super zeta function is defined for $\mathscr{R} s>1$ as,

$$
Z_{k}(s)=\prod_{\gamma \text { prim }} \prod_{q=0}^{\infty}\left(1-v_{\gamma}^{2 k} e^{-(s+q) l_{\gamma}}\right) \quad(k: \text { half integer }),
$$

and has the Selberg zeta function as its body. The only difference from the Selberg zeta function lies in the difference between the length spectrum of the super Fuchsian group with that of the Fuchsian group. Primitive lengths defined in (2.22) grow by matrix multiplication which is homogeneous, and using the property of the length spectrum of $\Gamma$ (see for instance, [24]),

$$
\begin{aligned}
n_{<l} & =\frac{1}{l} e^{l}(1+O(1)) \\
& =\{\text { number of primitive classes with lengths smaller than } l\},
\end{aligned}
$$

we see that $Z_{k}(s)$ is well defined and holomorphic in $s$ for $\mathscr{R} s>1$. (Holomorphicity is defined by the holomorphicity of all its coefficients in the expansion in terms of the nilpotents.)

We calculate the derivative of the super zeta function,

$$
\begin{aligned}
\left(\log Z_{k}(s)\right)^{\prime} & =\sum_{\gamma \text { prim }} \sum_{q=0}^{\infty} \frac{l_{\gamma} v_{\gamma}^{2 k} e^{-(s+q) l_{\gamma}}}{\left(1-v_{\gamma}^{2 k} e^{\left.-(s+q) l_{\gamma}\right)}\right.}=\sum_{\gamma \text { prim }} \sum_{q=0}^{\infty} \sum_{p=1}^{\infty} l_{\gamma} v_{\gamma}^{2 k p} e^{-(s+q) p l_{\gamma}} \\
& =\sum_{\gamma \text { prim }} \sum_{p=1}^{\infty} \frac{l_{\gamma} v_{\gamma}^{2 k p} e^{(1-s) l_{\gamma}}}{e^{p l_{\gamma}}-1} .
\end{aligned}
$$

Comparing above with the expression for $\zeta_{n}^{r}(s ; c)$ in (5.4), we derive,

$$
\begin{aligned}
\zeta_{n}^{r}(s ; c)= & \int_{0}^{\infty} d \lambda \frac{[\lambda(\lambda+2 c)]^{-s}}{\Gamma(s) \Gamma(1-s)} \frac{d}{d \lambda} \\
& \cdot\left\{\log \left[\frac{Z_{n}(n+\lambda+c+1)}{Z_{n+1 / 2}(n+\lambda+c+1 / 2)} \frac{Z_{n}(-n+\lambda+c)}{Z_{n+1 / 2}(-n+\lambda+c+1 / 2)}\right]\right\} .
\end{aligned}
$$


For generic values of $c$, we can integrate by parts to obtain,

$$
\frac{\partial \zeta_{n}^{r}}{\partial s}(0 ; c)=-\log \left[\frac{Z_{n}(n+c+1)}{Z_{n+1 / 2}(n+c+1 / 2)} \frac{Z_{n}(-n+c)}{Z_{n+1 / 2}(-n+c+1 / 2)}\right] .
$$

This together with (5.1), (5.2), (5.3) gives,

$$
\operatorname{sdet}\left(\Delta_{n}+c^{2}\right)=\left[\prod_{k=0}^{2|n|}\left(c^{2}-k^{2}\right)\right]^{-\mid \chi\left(M_{g} \mid / / 2\right.} \frac{Z_{n}(n+c+1)}{Z_{n+1 / 2}(n+c+1 / 2)} \frac{Z_{n}(-n+c)}{Z_{n+1 / 2}(-n+c+1 / 2)} .
$$

Analytically continuing $\operatorname{sdet}\left(\Delta_{n}+c^{2}\right)$ in $c$, and removing the zeros and possibly the nilpotents, we find,

$$
\operatorname{sdet} \Delta_{n}=\left[(2|n|) !(-1)^{n}\right]^{-\mid \chi\left(M_{g}||\right.} \frac{\tilde{Z}(n+1)}{\tilde{Z}(n+1 / 2)} \frac{\tilde{Z}(-n)}{\tilde{Z}(-n+1 / 2)},
$$

where $\tilde{Z}(m)=1 / r_{m} ! Z_{m}^{\left(r_{m}\right)}(m)$ is the leading coefficient of the Selberg super zeta function at a half integer point $m, r_{m}$ being the order of zero of its body at the point. We have assumed that the analytic continuation of the Selberg super zeta function exists for the appropriate half integer points. The order of zero of $\operatorname{sdet}\left(\Delta_{n}+c^{2}\right)$ at $c=0$ in (5.7) does not equal $\operatorname{dim} \operatorname{Ker} \Delta_{n}$. This is either an artifact of the analytic continuation, or suggests that the operator $\Delta_{n}$ is not diagonizable. We will use the notation sdet' instead of sdet when $\operatorname{Ker} \Delta_{n} \neq 0$.

Using the more general form of the Selberg trace formula than was used in Sect. 4, the ordinary Selberg zeta function can be shown to satisfy [24],

$$
\begin{aligned}
& \frac{1}{2 s-1} \frac{Z_{k}^{\prime}(s)}{Z_{k}(s)}-\frac{1}{2 \beta-1} \frac{Z_{k}^{\prime}(\beta)}{Z_{k}(\beta)} \\
& \quad=\sum_{n}\left[\frac{1}{(s-1 / 2)^{2}+r_{n}^{2}}-\frac{1}{(\beta-1 / 2)^{2}+r_{n}^{2}}\right]+2(g-1) \sum_{m=0}^{\infty}\left(-\frac{1}{s+m}+\frac{1}{\beta+m}\right) .
\end{aligned}
$$

After setting $\beta=1-s$, the nontrivial zeros $s=\frac{1}{2} \pm i r_{n}$ cancel and we can integrate with respect to $s$ to obtain the functional relation,

$$
Z_{k}(s)=x(s) Z_{k}(1-s)
$$

where

$$
x(s)=\exp \left(4 \pi(g-1) \int_{0}^{s-1 / 2} d v v \tan \pi v\right) .
$$

For fixed $s, x(s)$ depends only on the topology of the Riemann surface, and we will assume that this functional relation also holds for the super zeta function of the super Riemann surface, which has the same topology as its underlying Riemann surface. In particular, this would imply that the Selberg super zeta function has the same trivial zeros $s=-m(m \geqq 0, m$ integer $)$ including its order $(2 m+1) 2(g-1)$ as its body, the Selberg zeta function (cf. (5.10) below).

We use the functional relation (5.9) to reduce the formula (5.8) for the super 
determinants to a simpler form. Integrating by parts,

$$
\int_{0}^{s-1 / 2} d v v \tan \pi v=\frac{1}{\pi^{2}}\left[-\left.t \log \cos t\right|_{0} ^{(s-1 / 2) \pi}-L((s-1 / 2) \pi)\right]
$$

where $L(y)$ is the Lobachevsky function defined as in [25],

$$
L(y)=-\int_{0}^{y} d x \log \cos x
$$

and $L(s \pi)=s \pi \log 2$ for $s$ half integer. This allows us to calculate the leading behavior of $x(s)$ at half integer points as below. At integer points $s=m$,

$$
x(s) \stackrel{s \sim m}{\sim}(2 \pi(s-m))^{-2(g-1)(2 m-1)},
$$

and at half odd integer points $s=m+1 / 2$,

$$
x(m+1 / 2)=2^{-4 m(g-1)} .
$$

These together with the functional relation (5.9) determine the leading coefficient of $Z_{k}(s)$ at half integer points $\tilde{Z}(m)$ in terms of $\tilde{Z}(1-m)$ as follows:

$$
\begin{aligned}
\tilde{Z}(0) \tilde{Z}(1) & =(2 \pi)^{2(g-1)}\left(Z_{0}^{\prime}(1)\right)^{2}, \\
\tilde{Z}(-m) \tilde{Z}(1+m) & =(2 \pi)^{2(2 m+1)(g-1)}\left(Z_{0}(m+1)\right)^{2}, \\
\tilde{Z}(-m+1 / 2) \tilde{Z}(m+1 / 2) & =2^{4 m(g-1)}\left(Z_{1 / 2}(m+1 / 2)\right)^{2}, \\
m & =1,2,3, \ldots
\end{aligned}
$$

These reduce the super determinants in (5.8) to the following:

Integer spin

Half integer spin

$$
\begin{aligned}
\operatorname{sdet}^{\prime} \Delta_{0} & =(2 \pi)^{2(g-1)}\left(\frac{Z_{0}^{\prime}(1)}{\widetilde{Z}(1 / 2)}\right)^{2} \\
\operatorname{sdet}^{\prime} \Delta_{-1} & =\left(\frac{\pi}{4}\right)^{2(g-1)}\left(\frac{Z_{0}^{\prime}(1)}{Z_{1 / 2}(3 / 2)}\right)^{2} \\
\operatorname{sdet} \Delta_{n} & =\left(\frac{2 \pi^{2 n+1}}{(2 n) !}\right)^{2(g-1)}\left(\frac{Z_{0}(n+1)}{Z_{1 / 2}(n+1 / 2)}\right)^{2} \\
n & =1,2,3, \ldots \\
\operatorname{sdet}^{\prime} \Delta_{-n} & =\left(\frac{\pi^{2 n-1}}{(2 n) ! 2}\right)^{2(g-1)}\left(\frac{Z_{0}(n)}{Z_{1 / 2}(n+1 / 2)}\right)^{2} \\
n & =2,3,4, \ldots .
\end{aligned}
$$$$
\operatorname{sdet}^{\prime} \Delta_{1 / 2}=(-1)^{g-1}\left(\frac{2}{\pi}\right)^{2(g-1)}\left(\frac{Z_{1 / 2}(3 / 2)}{Z_{0}^{\prime}(1)}\right)^{2}
$$$$
\operatorname{sdet}^{\prime} \Delta_{-1 / 2}=(-1)^{g-1}(2 \pi)^{-2(g-1)}\left(\frac{\tilde{Z}(1 / 2)}{Z_{0}^{\prime}(1)}\right)^{2}
$$ 


$$
\begin{aligned}
\operatorname{sdet} \Delta_{n-1 / 2} & =(-1)^{g-1}\left(\frac{2}{(2 n-1) ! \pi^{2 n-1}}\right)^{2(g-1)}\left(\frac{Z_{1 / 2}(n+1 / 2)}{Z_{0}(n)}\right)^{2} \\
n & =2,3,4, \ldots
\end{aligned}
$$

$$
\operatorname{sdet}^{\prime} \Delta_{-n-1 / 2}=(-1)^{g-1}\left((2 n+1) ! 2 \pi^{2 n+1}\right)^{-2(g-1)}\left(\frac{Z_{1 / 2}(n+1 / 2)}{Z_{0}(n+1)}\right)^{2},
$$

$$
n=1,2,3, \ldots
$$

where

$$
\tilde{Z}(1 / 2)=\frac{1}{r_{1 / 2} !} Z_{1 / 2}^{\left(r_{1 / 2}\right)}(1 / 2)
$$

The superstring measure for genus $g \geqq 2$ super Riemann surfaces with compact body is $[2,3]$,

$$
\begin{aligned}
Z_{g} & =\int_{s \mathscr{M}_{g}} d(\mathrm{SWP})\left(\frac{\operatorname{sdet}^{\prime} \Delta_{0}}{\int_{s M_{g}} E}\right)^{-5 / 2}\left(\operatorname{sdet} \Delta_{1}\right)^{1 / 2} \\
& =\left(32 \pi^{2}\right)^{-(g-1)} \int_{s \mathscr{M}_{g}} d(\mathrm{SWP}) \frac{\tilde{Z}^{5}(1 / 2)}{Z_{0}^{\prime 5}(1)} \frac{Z_{0}(2)}{Z_{1 / 2}(3 / 2)},
\end{aligned}
$$

where $s \mathscr{M}_{g}$ is the supermoduli space for genus $g$ surfaces and $d(\mathrm{SWP})$ is the super Weil-Petersson measure on $s \mathscr{M}_{g}$.

\section{Appendix A}

In this appendix, we outline the calculation of $h_{n}^{t}(r)$ in (3.14). Define,

$$
\begin{aligned}
f_{1}(b) & =e^{-b^{2} / 4 t} \\
f_{2}(b, r) & =\frac{\sinh (n+1 / 2) b}{\sqrt{\cosh b-\cosh r}} T_{2 n}\left(\frac{\cosh b / 2}{\cosh r / 2}\right),
\end{aligned}
$$

so that (3.13),

$$
g_{n}^{t}(r)=\frac{-1}{2 \pi^{3 / 2} t^{1 / 2} \sqrt{2}} \int_{r}^{\infty} d b f_{1} f_{2} .
$$

Also define the function $F(r, b)$ say,

Using

$$
\frac{2(2 n+1)}{\sinh r} h_{n}^{t}(r)=\frac{1}{2 \pi^{3 / 2} t^{1 / 2} \sqrt{2}} \int_{r}^{\infty} d b F(r, b) .
$$

and (3.6), we derive

$$
\frac{\partial}{\partial t}\left(t^{-1 / 2} f_{1}\right)=\frac{\partial^{2}}{\partial b^{2}}\left(t^{-1 / 2} f_{1}\right)
$$

$$
\begin{aligned}
F(r, b) & =\left(\frac{\partial}{\partial b} f_{1}\right)\left[2\left(\frac{\partial}{\partial b}+\frac{\partial}{\partial r}\right)+\left(\frac{1}{\sinh r}-2 n \tanh \frac{r}{2}\right)\right] f_{2} \\
& +f_{1}\left[\left(\frac{\partial}{\partial b}+\frac{\partial}{\partial r}\right)^{2}+\left(\frac{1}{\sinh r}-2 n \tanh \frac{r}{2}\right)\left(\frac{\partial}{\partial b}+\frac{\partial}{\partial r}\right)+n^{2}\left(1+\frac{2}{\cosh r+1}\right)\right] f_{2}
\end{aligned}
$$


To integrate by parts with respect to $b$, we introduce a function $H(r, b)$ (yet to be determined) such that,

$$
\left[2\left(\frac{\partial}{\partial b}+\frac{\partial}{\partial r}\right)+\left(\frac{1}{\sinh r}-2 n \tanh \frac{r}{2}\right)\right] f_{2}-H \stackrel{b \rightarrow r}{\longrightarrow} 0
$$

so that

$$
F(r, b)=\frac{\partial}{\partial b}\left\{f_{1}\left[2\left(\frac{\partial}{\partial b}+\frac{\partial}{\partial r}\right)+\left(\frac{1}{\sinh r}-2 n \tanh \frac{r}{2}\right)\right] f_{2}-f_{1} H\right\}+f_{1} K+\frac{\partial}{\partial b} f_{1} H
$$

where $K(r, b)$ is defined as,

$$
K(r, b)=\left[\left(\frac{\partial^{2}}{\partial r^{2}}-\frac{\partial^{2}}{\partial b^{2}}\right)+\left(\frac{1}{\sinh r}-2 n \tanh \frac{r}{2}\right)+n^{2}\left(1+\frac{2}{\cosh r+1}\right)\right] f_{2}+\frac{\partial}{\partial b} H
$$

The surface term does not contribute to $h_{n}^{t}(r)$ due to the condition (A1). The choice,

$$
H(r, b)=\frac{(2 n+1)}{\sqrt{\cosh b-\cosh r}} \frac{\cosh n b}{\cosh r / 2} T_{2 n+1}\left(\frac{\cosh b / 2}{\cosh r / 2}\right)
$$

satisfies (A1) and gives,

$$
K(r, b)=\frac{2 n(2 n+1)}{\sqrt{\cosh b-\cosh r}} \frac{\sinh (n+1 / 2) b}{(\cosh r+1)} T_{2 n}\left(\frac{\cosh b / 2}{\cosh r / 2}\right) .
$$

We have made use of the following properties of Chebyshev polynomials.

$$
\begin{aligned}
& \left(x^{2}-1\right) T_{2 n}(x)^{\prime \prime}+x T_{2 n}(x)^{\prime}-4 n^{2} T_{2 n}(x)=0, \\
& T_{2 n+1}(x)=x T_{2 n}(x)+\frac{\left(x^{2}-1\right)}{2 n} T_{2 n}^{\prime}(x), \\
& T_{2 n+1}^{\prime}(x)=(2 n+1) T_{2 n}(x)+\left(\frac{2 n+1}{2 n}\right) x T_{2 n}^{\prime}(x) .
\end{aligned}
$$

This finally gives $h_{n}^{t}(r)$ as in (3.14). $g_{n}^{t}(r)$ and $h_{n}^{t}(r)$ can be shown to satisfy (3.7) in a similar manner.

\section{Appendix B}

In this appendix we calculate integrals of the following form,

where

$$
J_{k}, P_{k}, K_{k}, N_{k}, N_{k}^{\prime}=\int_{-v_{0}}^{v_{0}} \frac{d v}{\sqrt{v_{0}^{2}-v^{2}}}\left(\frac{\cosh \frac{p l_{\gamma}}{2}+i v \sinh \frac{p l_{\gamma}}{2}}{\cosh \frac{p l_{\gamma}}{2}-i v \sinh \frac{p l_{\gamma}}{2}}\right)^{k / 2} T_{k}\left(\frac{\cosh b / 2}{\cosh r / 2}\right) F(v)
$$

$$
v_{0}=\frac{\sqrt{\cosh b-\cosh p l_{\gamma}}}{\sqrt{2} \sinh \frac{p l_{\gamma}}{2}}
$$


and the functions $F(v)$ are defined as follows.

$$
\begin{array}{ll}
J_{k} & 1 \\
P_{k} & i v \\
K_{k} & v^{2} \\
N_{k} & \frac{1}{\cosh \frac{p l_{\gamma}}{2}+i v \sinh \frac{p l_{\gamma}}{2}} \\
N_{k}^{\prime} & \frac{1}{\cosh \frac{p l_{\gamma}}{2}-i v \sinh \frac{p l_{\gamma}}{2}} .
\end{array}
$$

For example,

$$
N_{2 n}=\int_{-v_{0}}^{v_{0}} \frac{d v}{\sqrt{v_{0}^{2}-v^{2}}}\left(\frac{\cosh \frac{p l_{\gamma}}{2}+i v \sinh \frac{p l_{\gamma}}{2}}{\cosh \frac{p l_{\gamma}}{2}-i v \sinh \frac{p l_{\gamma}}{2}}\right)^{n} \frac{T_{2 n}\left(\frac{\cosh b / 2}{\cosh r / 2}\right)}{\cosh \frac{p l_{\gamma}}{2}+i v \sinh \frac{p l_{\gamma}}{2}} .
$$

As in [8], we can calculate the generating functions for the above integrals by making use of the following generating function for the Chebyshev polynomials and residue calculus:

$$
\sum_{k=0}^{\infty} \lambda^{k} T_{k}(x)=\frac{1-\lambda x}{1-2 \lambda x+\lambda^{2}}
$$

We define the generating functions for the integrals as,

$$
J=\sum_{k=0}^{\infty} \lambda^{k} J_{k}, \quad \text { etc. }
$$

Defining $x_{0}=\cosh b / 2 / \cosh p l_{\gamma} / 2$, we derive the following results:

$$
\begin{aligned}
& J=\pi \frac{1}{1-\lambda} \\
& P=-\pi \frac{\cosh \frac{p l_{\gamma}}{2}}{\sinh \frac{p l_{\gamma}}{2}}\left(x_{0}-1\right) \frac{\lambda}{(1-\lambda)^{2}} \\
& K=\pi \frac{\cosh ^{2} \frac{p l_{\gamma}}{2}}{\sinh ^{2} \frac{p l_{\gamma}}{2}}\left[\frac{(1+\lambda)}{(1-\lambda)^{3}}\left(x_{0}-1\right)+\frac{(1-3 \lambda)}{2(1-\lambda)^{3}}\left(x_{0}^{2}-1\right)\right]
\end{aligned}
$$




$$
\begin{aligned}
N & =\pi \frac{1}{\cosh \frac{p l_{\gamma}}{2}} \frac{1}{x_{0}\left(1-\lambda x_{0}\right)} \\
N^{\prime} & =\pi \frac{1}{\cosh \frac{p l_{\gamma}}{2}} \frac{1}{x_{0}-\lambda} .
\end{aligned}
$$

Acknowledgements. The author would like to thank Eric D'Hoker for suggesting this problems, for numerous discussions, and for a careful reading of the manuscript.

\section{References}

1. Polyakov, A. M.: Quantum geometry of bosonic strings. Phys. Lett. 103B, 207-210 (1981); Quantum geometry of fermionic strings. Phys. Lett. 103B, 211-213 (1981)

2. D’Hoker, E., Phong, D.: Superholomorphic anomalies and supermoduli space. Nucl. Phys. B292, 317-329 (1987).

3. D'Hoker, E., Phong, D.: The geometry of quantized strings. to be published in Rev. Mod. Phys.

4. Chaudhuri, S., Kawai, H., Tye, S. H.: Path-integral formulation of closed strings. Phys. Rev. D15, 1148-1168 (1987)

5. Baranov, M. A., Schwarz, A. S.: Multiloop contribution to string theory. Pis'ma Zh. Exsp. Teor. Fiz. 42, 340-342 (1985)

6. D'Hoker, E., Phong, D.: Loop amplitudes for the fermionic string. Nucl. Phys. B278, 225-241 (1986)

7. Moore, G., Nelson, P., Polchinski, J.: Strings and supermoduli. Phys. Lett. 169B, 47-53 (1986)

8. D'Hoker, E., Phong, D.: On determinants of Laplacians on Riemann surfaces. Commun. Math. Phys. 104, 537-545 (1986)

9. Kierlanczyk, M.: MIT Ph. D. thesis.

10. Sarnak, P.: Determinants of Laplacians. Commun. Math. Phys. 110, 113-120 (1987)

11. Fay, J. D.: Fourier coefficients of the resolvent for a Fuchsian group. J. Reine. Angew. Math. 293, 143-203 (1977)

12. Baranov, A. M., Manin, Yu. I., Frolov, I. V., Schwarz, A. S.: A Superanalog of the Selberg trace formula and multiloop contributions for fermionic strings. Commun. Math. Phys. 111, 373-392 (1987); The multiloop contribution in the fermion string. Yad. Fiz. 43, 1053-1056 (1986)

13. Martinec, E. J.: Conformal field theory on a (super-)Riemann surface. Nucl. Phys. B281, 157-210 (1987).

14. Baranov, M. A., Frolov, I. V., Schwarz, A.S.: Geometry of two-dimensional superconformal field theories. Teor. Mat. Fiz. 70, 92-103 (1987)

15. Crane, L., Rabin, J.: Super Riemann surfaces: Uniformization and Teichmüller Theory. Commun. Math. Phys. 113, 601-623 (1988); Supermanifolds and super Riemann surfaces. EF186-56; University of Chicago preprint

16. DeWitt, B.: Supermanifolds. Cambridge: Cambridge University Press 1984

17. Howe, P. S.: Super Weyl transformations in two dimensions. J. Phys. A12, 393-402 (1979)

18. Moore, G., Nelson, P.: Heterotic geometry. Nucl. Phys. B274, 509-519 (1986)

19. van Nieuwenhuizen, P.: Supergravity. Phys. Rep. C68, 189-398 (1981), appendix F

20. Friedan, D.: Notes on string theory and two dimensional conformal field theory. In Unified string theories. Singapore: World Scientific 1986

21. Martinec, E.: Superspace geometry of fermionic strings. Phys. Rev. D15, 2604-2613 (1983)

22. Selberg, A.: Harmonic analysis and discontinuous groups in weakly symmetric Riemannian spaces with applications to Dirichlet series. J. Indian Math. Soc. 20, 47-87 (1956)

23. Fried, D.: Analytic torsion and closed geodesics on hyperbolic manifolds. Invent. Math. 84, 523-540 (1986) 
24. Hejhal, D. A.: The Selberg trace formula for $\operatorname{PSL}(2, R)$. Lecture Notes in Mathematics, Vol 548; Berlin Heidelberg, New York: Springer 1976; Duke Math. J. 43, 441-482 (1976)

25. Gradshteyn, I. S., Ryzhik, I. M.: Table of integrals, series and products. 8.26. New York: Academic Press 1965

26. Gradshteyn, I. S., Ryzhik, I. M.: Table in integrals, series and products. 9.225. New York: Academic Press 1965

27. Gradshteyn, I. S., Ryzhik, I. M.: Table of integrals, series and products. 9.111. New York: Academic Press 1965

28. Ray, D. B., Singer, I. M.: Analytic torsion for complex manifolds. Ann. Math. 98, 154-177 (1973)

Communicated by S.-T. Yau

Received November 16, 1987 
